\title{
Revisión de experiencias urbanas y periurbanas de cría animal como alternativa de seguridad alimentaria
}

\section{Review of urban and suburban experiences of animal breeding as an alternative of food security}

\author{
Camilo Ernesto Seija Flórez \\ E-mail: cseija@gmail.com \\ Universidad Nacional Abierta y a Distancia (UNAD) \\ Escuela de Ciencias Agrícolas, Pecuarias y del Medio Ambiente (ECAPMA)
}

Resumen.- La producción animal urbana y periurbana en países tropicales constituye una estrategia válida para mejorar la nutrición humana, el autoconsumo familiar, la generación de ingresos, el ahorro y las condiciones de vida. Mediante estudios de caso se revisaron experiencias de cría animal (porcícola, acuícola, de leche, avícola, cunícola y apícola) en algunas ciudades del mundo para contribuir a resolver problemas de abastecimiento de alimentos en áreas urbanas y periurbanas. Se destacan las limitaciones (políticas, legales, sanitarias y ambientales) y las estrategias de intervención buscando soluciones a los problemas. Se concluyó que a partir de las experiencias positivas alrededor del mundo, es posible introducir consideraciones de sostenibilidad en la crianza animal urbana y periurbana.

Palabras clave: Agricultura urbana y periurbana, desarrollo local, producción animal urbana y periurbana, producción familiar, seguridad alimentaria.

Abstract.- The urban and suburban animal production in tropical countries is a valid strategy to improve human nutrition, family consumption, income generation, savings and living conditions. Through case studies experiences of breeding animal (pigs, aquaculture, dairy, poultry, rabbits and bees) were reviewed in some cities around the world to help in solving problems of food supply in urban and suburban areas. It should highlight the constraints (political, legal, health and environmental) and intervention strategies to find solutions to problems. It was concluded that the positive experiences from around the world can introduce considerations of sustainability in urban and suburban animal breeding.

Key words: urban agriculture, local development, urban and suburban animal production, household production, food security. 


\section{Introducción}

En las últimas décadas las ciudades del mundohan experimentado un crecimiento poblacional acelerado debido a la alta natalidad y la disminución progresiva de la mortalidad -características de países en vía de desarrollo- y al desplazamiento forzado de la población rural a los centros urbanos debido a la falta de oportunidades y a la guerra que azota las zonas rurales. Castro et al (2002) afirman que la población en condiciones de desplazamiento llega a áreas socioeconómicamente deprimidas de las ciudades, donde la vulnerabilidad es muy marcada, creando focos de pobreza que se caracterizan, entre otros aspectos, por la informalidad en las modalidades de empleo.

Con mayor población y menores oportunidades de empleo se hace más visible la carencia de alimentos para satisfacer las necesidades nutricionales, viéndose afectada la seguridad alimentaria de la población. Por este motivo, el gobierno y las agencias de cooperación internacional han diseñado estrategias para que las poblaciones urbanas y periurbanas puedan producir sus propios alimentos, creándose así sistemas de agricultura y producción pecuaria en áreas urbanas y periurbanas.

Para el caso de Colombia, las actividades de agricultura urbana y periurbana se han dedicado a transferir principalmente tecnologías agrícolas. Por esta razón, los proyectos de seguridad alimentaria deben integrar la dieta proteínica generada por los sistemas pecuarios en áreas urbanas y periurbanas cuyas poblaciones carecen de recursos económicos suficientes.

Por lo anterior, la investigación se propuso revisar estudios de caso de cría animal urbana y periurbana en distintas ciudades del mundo, con el fin de enriquecer los enfoques de los proyectos de desarrollo financiados en Colombia por Organizaciones no gubernamentales, Universidades, Instituciones del estado y empresas privadas.

\section{Metodología}

La investigación se basa en estudios de caso de producción agrícola, porcícola, acuícola, de leche, avícola, cunícola y apícola desarrollada en áreas urbanas y periurbanas de diferentes ciudades del mundo que han ayudado a resolver problemas de abastecimiento de alimentos, generación de ingresos, empleo y mejor calidad de vida dentro de las poblaciones. También documenta las limitaciones (políticas, legales, sanitarias y ambientales) y las estrategias de intervención en busca de soluciones a los problemas.

\section{Resultados y discusión}

\section{La agricultura urbana y periurbana}

Según IPES (2006), la agricultura urbana y periurbana debe realizarse buscando la producción o transformación en forma inocua de los alimentos de autoconsumo o comercialización; debe aprovechar eficiente y sosteniblemente los recursos e insumos locales respetando saberes y conocimientos locales; debe promover la equidad de género a través del uso 
de tecnologías apropiadas y procesos participativos y mejorar la calidad de vida de la población urbana y la gestión urbana social y ambientalmente sustentable de las ciudades.

Esto implica la diversidad en cultivos y en crianza animal para fines alimenticios. Significa sembrar plantas aromáticas, medicinales y ornamentales y sacar productos derivados de árboles; incluye la producción de insumos (plántulas, compost, humus), la transformación y comercialización de productos agrícolas y pecuarios y los programas de sanidad animal.

Este tipo de actividad comprende modalidades organizativas (individual, grupal o por empresas de pequeña, mediana y gran escala), puede llevarse a cabo en terrenos privados, públicos o institucionales, usa mano de obra de residentes urbanos y periurbanos, emplea recursos urbanos (residuos orgánicos, aguas residuales), establece vínculos directos con los consumidores y es parte del sistema urbano de alimentos.

Para Drescher (2001) esta actividad puede ser parte de políticas de seguridad alimentaria o de reducción de la pobreza y de políticas de desarrollo local (generación de ingresos y empleos para lograr ciudades productivas). Igualmente se inscribe dentro de las estrategias de seguridad alimentaria que la FAO (2001) define cuando todas las personas tienen en todo momento acceso material y económico a suficientes alimentos inocuos y nutritivos para satisfacer sus necesidades alimenticias y las preferencias alimenticias a fin de llevar una vida activa y sana.

\section{Porcicultura urbana y periurbana}

En un estudio realizado en Cuba, Casanova et al (2006) encontraron que las razas utilizadas son Duroc, Landrace y Hampshire. Las ganancias medias diarias de peso promedio fueron de 384,9 gr para el grupo de los campesinos, 434 gr para los independientes y 461 gr para los obreros. Los mayores valores se encontraron en el grupo de productores especializados con 637 gr, ya que la alimentación de los animales en este grupo consiste en concentrados comerciales, mientras que en los demás grupos se basa en desechos de comida.

Para evitar el contagio de enfermedades, los productores que alimentan sus animalescondesechosdecomida cocinan los alimentos antes de suministrárselos. Las enfermedades más frecuentes son respiratorias y gastrointestinales, como la disentería porcina (Serpulina hyodysenteriae), de transmisión fecaloral en heces de perros, ratas y ratones que viven en explotaciones porcinas. La reproducción se realiza de forma natural a excepción de los reproductores que viven cerca de centros especializados de porcicultura que hacen uso de la inseminación artificial.

Arce et al (2005) encontraron en el distrito de Lurigancho Chosica, Lima (Perú) que la mayoría de beneficiarios con el proyecto de agricultura urbana son hombres de escolaridad primaria que consideran la actividad como generadora de los ingresos para sobrevivir, en tanto que otros consideran que solo constituye un ahorro complementario. Los productores se especializan en vender cerdos de dos meses para pie de 
cría y de cuatro meses para engorde, utilizan para alimentación los residuos de restaurantes, casas, mercados y panaderías, y las vísceras de pollo de granjas avícolas. Las razas no son puras sino producto de cruces de animales criollos con razas como Landrace, Pietrán, y Duroc. La reproducción es a través de monta natural en el $85 \%$ de los casos.

La investigación de la Facultad de Veterinaria de la Universidad de la República y una Organización no gubernamental en asentamientos urbanos y periurbanos de Montevideo (Uruguay), reporta que los productores del proyecto utilizan sistemas un tanto intensivos y de baja inversión, con restos de cocina, panadería y subproductos de los mataderos. Estos alimentos son suministrados sin tratarlos, pues solo en algunos casos los productores los cocinan. Ante este problema, el grupo encargado del proyecto, integrado por médicos veterinarios, desarrolló una técnica de fermentación controlada, aplicando melaza y levadura proteolítica (Hansenula Montevideo) (Castro, 2007).

Según Santandreu et al (2006), se redujo el $\mathrm{pH}$ con desaparición total de Escherichia coli. A los 15 días de iniciado el proceso, los cerdos aceptaron sin dificultad el alimento y no registraron síntomas clínicos de enfermedad. A partir de esta investigación se permitió la utilización de la levadura proteolítica en el tratamiento de los alimentos para cerdos en las áreas urbanas y periurbanas de Montevideo, Uruguay.

En Hubli Dharward (India) por tradición, un número significativo de cerdos deambulan por las calles, y su fuente alimentaria son las basuras y restos de comida de restaurantes, resultando estos animales una fuente de proteína barata para sus habitantes (Nunan, 2005). Las autoridades públicas recomiendan su encierro ante la amenaza para la salud pero los propietarios se resisten porque representa mano de obra adicional y retrasos en el crecimiento, además de que en el mercado existe la concepción de que merma la calidad si el animal ha estado encerrado.

Uno de los principales problemas es el tratamiento de las excretas, no obstante la utilización como abono debidamente compostado. Otro problema es la contaminación ambiental por la baja digestibilidad del fósforo en las dietas de los cerdos, lo que exige un tratamiento especial.Las soluciones planteadas radican en la mayor eficiencia del fósforo, que al tratarse con enzimas fitasas contribuye a disminuir la excreción al ambiente.

\section{Acuicultura urbana y periurbana}

Cuba es uno de los países que más ha incentivado la acuicultura familiar con el propósito de mejorar la disponibilidad de alimentos. Una o varias familias utilizan estanques en concreto o tierra en el patio para criar carpa común (Cyprinus carpio), carpa herbívora (Ctenophayngodon idellus), o carpa colosoma (Colosoma macropomum) (Coto, 2005). Estas especies son las recomendadas porque consumen alimento del agua y los mismos productores las nutren de forma artesanal.

Las aguas lluvias se colectan del techo de las casas, que luego de usarse en policultivos, riegan huertos y árboles 
frutales. Una de las especies más cultivadas con sistemas de recirculación y en estanques de 1 a $50 \mathrm{~m} 3$ es el bagre; los costos de la tierra, los robos, la expansión urbana (doméstica e industrial) y la contaminación por plomo son los problemas que enfrenta la acuicultura urbana de estos lugares.

Según Khov et al (2005), en la ciudad de Phnom Penh (Camboya) existen pantanos donde hay peces y vegetales acuáticos comestibles, que son fuente de alimentación humana.

\section{Producción láctea}

Mekasha et al (2003) encontraron que entre las diversas formas de producción lechera tropical y subtropical se encuentran los sistemas urbanos y periurbanos de producción, procesamiento y mercado de leche y productos lácteos que se ofrecen a los consumidores. En ciudades secundarias etíopes existen granjas especializadas que producen leche y mantequilla para los mercados locales.

Algunos de estos sistemas de producción llegan a ser muy eficientes ya que los productores tienen conocimientoy técnicas de nutrición, genética, sanidad y manejo en general. En Addis Abeba, capital de Etiopía, el estudio de caracterización de los sistemas de producción lechera financiado por el Instituto internacional de investigación ganadera encontró que en los sistemas periurbanos no se ofrece alimentación balanceada con los residuos de cosechas, de cervecerías y de aves.

En las zonas urbanas y periurbanas de producción lechera se utiliza el ganado puro, en especial el Friesan, y los búfalos. En Hubli Dharwad se emplean para la producción de leche especialmente los búfalos. La posesión de estos en las familias es tradición, y por eso en las áreas urbanas y periurbanas de la ciudad existen grandes lecherías. En Colombia es común que en áreas periurbanas haya propietarios de grandes ganaderías de leche y carne que generen un desarrollo muy marcado, en una actividad que contribuye a la seguridad alimentaria y a la generación de empleo e ingresos.

\section{Avicultura}

Uno de los sistemas productivos más utilizados es la cría de pollos y gallinas para carne y huevos. En Córdoba (Argentina) entre 1994 y 1995 la localidad se hundió en una profunda crisis económica, agravada por la quiebra de la cooperativa agrícola-ganadera San Martín y el cierre de dos asociaciones locales prestadoras de dinero. Ante esta situación, las autoridades municipales movilizaron recursos a la comunidad para transformar un escenario negativo en uno con grandes posibilidades (Silvana, 2006).

El objetivo de este proyecto fue contribuir a la seguridad alimentaria de las familias más vulnerables de la región, para lo cual se utilizaron terrenos privados de familias o lotes baldíos en zonas urbanas y periurbanas y se organizaron grupos de familias que recibieron crédito, capacitación y asistencia técnica y social. El proyecto permitió acrecentar las relaciones entre familias beneficiarias, el municipio, las instituciones de bien público y la comunidad (Silvana, 2006). 
La cría de pollos es una actividad practicada en áreas urbanas y periurbanas de Colombia que aporta a la seguridad alimentaria de la población y que debe ser más apoyada por las instituciones, con técnicas de producción específicamente en lo relacionado con la nutrición, con el propósito de disminuir los costos de producción.

\section{Apicultura}

La apicultura no se realiza solo en zonas rurales, sino también se está realizando en terrazas y edificios de ciudades. Entre las ciudades que practican la apicultura está Tokio. Algunos centros comerciales tienen en las terrazas colmenas que llegan a producir anualmente alrededor de 300 $\mathrm{Kg}$ de miel. Las abejas se alimentan de flores de árboles de castaño que se encuentran en las afueras de la ciudad.

En Francia desde hace tres años se desarrolla un programa de recuperación de colmenas urbanas. París está poblada por abejas de 300 colmenas que han colonizado techos, balcones, parques, centros comerciales, museos y hoteles. Por ejemplo, el hotel Eiffel Park produce en tres colmenas 150 kilos de miel al año, un producto que sus clientes degustan en el desayuno y en deliciosos postres (Piedad, 2009).

La Asociación francesa de apicultores, que fomenta el cultivo urbano en ciudades como Nantes, Paris y Lille, explica que es también una forma de llamar la atención sobre la importancia de los ecosistemas de estos insectos y su situación cada vez más crítica.

No sucede de igual forma con los apicultores clandestinos de New York, dado que la legislación vigente no permite criar animales feroces, salvajes o peligrosos en la ciudad, incluidas las abejas. Pocos apicultores admiten que se dedican a esta actividad porque la multa es de 2000 dólares.

En Alemania hay preocupación por la disminución de la población de abejas, debido a que los expertos han determinado que esta reducción puede tener serias consecuencias sobre la polinización de frutales. Los apicultores de Wiendenhoft han trabajado arduamente para atraer gente joven a esta profesión que de momento incluye un porcentaje alto de personas mayores. Ahora cientos de miles de abejas están constantemente en los cielos de ciudades como Frankfurt, Múnich y Hamburgo; esta última tiene al menos 50 millones de abejas en 2100 colmenas. En la sola capital alemana existen unos 500 apicultores.

Uno de los casos positivos de apicultura urbana se experimenta en Inglaterra donde la Asociación inglesa de apicultores tiene al menos 5000 miembros registrados que crían abejas en los patios y tejados de la ciudad de Londres.

Pero no solo es un pasatiempo para las ciudades desarrolladas. En la República Democrática del Congo se han definido programas de agricultura urbana como una manera de que la población acceda a alimentos frescos y plantas medicinales que fortalezcan su sistema inmunológico. Aquí la miel, además de deliciosa, resulta interesante por sus propiedades medicinales sobre todo en países con pocos recursos económicos. 


\section{Cunicultura}

La producción de conejos y cuyes se convierte en una opción interesante en áreas urbanas y periurbanas debido a los espacios reducidos que necesita. En Lima (Perú) la producción de cuyes (Cavia porcellus) se convirtió en una alternativa viable, generadora de ingresos y fuente alimenticia para la familia, labor que es realizada principalmente por las mujeres del hogar.

Según Francia \& Bautista (2005), la experiencia productiva de los cuyes se desarrolló de la siguiente manera: Se capacitóla comunidadbeneficiariateniendo en cuenta que esta población ya tenía cría de cuyes en sus patios, se entregaron reproductores y se instruyó sobre la alimentación, a base de forraje restringido, trabajo que duplicó la producción.

La comercialización de los cuyes se realizó a través de una asociación que exigía normas de calidad a cada uno de sus integrantes, con el fin de mantener el prestigio ganado a través del tiempo. La parte de la producción que no es vendida es utilizada en la familia para el autoconsumo, lo que promueve la seguridad alimentaria del hogar y además, genera ahorro en la compra de proteína de origen animal. Los productores de cuyes se están agrupando de manera eficiente, lo que les permite comprar el concentrado al por mayor más barato y producir forrajes para sus animales.

Según Francia \& Bautista (2003), el $44 \%$ de los productores manifiestan vender los animales vivos, el $17 \%$ sacrificados, el 6\%preparados y $33 \%$ a los intermediarios. Otros proyectos cunícolas han salido avante por diversos factores como la guerra en el sector oriental de la República Democrática del Congo, que duró desde 1996 hasta 2003 y que causó una fuerte disminución del ganado.

En Colombia la cuyicultura y la cunicultura sonactividades productivas desconocidas, lo cual se refleja en el bajo consumo per cápita (3\%), a pesar de que la carne de conejo es una de las más representativas en cuanto a calidad. No se la puede dejar por fuera de este análisis ya que es una alternativa viable para espacios reducidos de áreas urbanas y periurbanas. Además, las excretas son de fácil manejo y una alternativa para la fertilización de los cultivos que aplican técnicas de agricultura orgánica, por sus altos niveles de materia orgánica, nitrógeno y potasio (Tabla1).

Tabla 1. Composición media del estiércol de diferentes animales domésticos

\begin{tabular}{lccccc}
\hline \multicolumn{1}{c}{ Nutriente } & $\%$ & Vacunos & Porcinos & Conejos & Gallinas \\
\hline Materia orgánica & $\%$ & 48,9 & 45,3 & 63,9 & 54,1 \\
Nitrógeno total & $\%$ & 1,27 & 1,36 & 1,94 & 2,38 \\
Fósforo asimilable (P2 05) & $\%$ & 0,81 & 1,98 & 1,82 & 3,86 \\
Potasio (K2O) & $\%$ & 0,84 & 0,66 & 0,95 & 1,39 \\
Calcio (CaO) & $\%$ & 2,03 & 2,72 & 2,36 & 3,63 \\
Magnesio (MgO) & $\%$ & 0,51 & 0,65 & 0,45 & 0,77 \\
\hline
\end{tabular}


Si se tiene en cuenta los datos de la Tabla 1 , puede decirse que la incorporación de $20.000 \mathrm{~kg} / \mathrm{ha}$ de abono orgánico a base de ganado vacuno aportan al suelo $50,8 \mathrm{~kg}$ de N, 33,8 $\mathrm{kg}$ de $\mathrm{K}$ y 32,4 $\mathrm{kg}$ de fósforo asimilable; mientras que igual dosis de gallinaza aporta $142,8 \mathrm{~kg}$ de N, 83, $\mathrm{kg}$ de $\mathrm{K}$ y $231,6 \mathrm{~kg}$ de fósforo asimilable.

También hay que considerar que la agricultura orgánica además de cuidar la salud de los consumidores, en algunos casos agrega valor a algunos productos. Por ejemplo, en las plantas aromáticas la calidad del ingrediente activo es reconocido por la industria farmacéutica cuando se utilizan abonos orgánicos, mientras que los fertilizantes químicos la desmejoran (Farmaverde, 2006).

\section{Problemas en la producción de animales}

\subsection{Problemática legal y política}

En la mayoría de los casos los productores se encuentran en una situación de ilegalidad, ya que la cría y comercialización de animales es una actividad prohibida o no contemplada en los planes de ordenamiento territorial o desarrollo urbano (Castro et al, 2002). Incluso así, los gobiernos suelen tolerar esta actividad, teniendo en cuenta la situación de empobrecimiento progresivo de algunas poblaciones involucradas en esta actividad.

Debe examinarse los problemas de los sistemas de producción, estudiando el impacto de la agricultura urbana en la salud y evaluando los peligros de la cría de ganado y el riesgo de las enfermedades zoonóticas. Con una buena legislación se puede trazar una reglamentación que controle la actividad y proteja la salud de los residentes y el medio ambiente de las ciudades.

\subsection{Problemática sanitaria}

La cría animal con residuos orgánicos aumenta la aparición de un gran número de enfermedades zoonóticas, riesgo que se corre cuando los animales son criados cerca de las viviendas, -bien sea por no disponer de espacio o para tener control sobre ellos-, o cuando se utilizan residuos sin tratamiento para su alimentación o cuando no hay oportunidad para acceder a residuos orgánicos debidamente tratados. Ante algunas carencias en la gestión integral municipal de los residuos y en el asesoramiento técnico profesional, agravadas por la situación de pobreza e ilegalidad, muchos productores no acuden a un técnico particular, servicio que con frecuencia ofrecen los programas de extensión de las organizaciones sociales regionales.

\subsection{Problemática ambiental}

La manipulación de residuos en un espacio reducido y próximo a las casas impacta el ambiente, el terreno, los cursos de agua y las vías públicas, al dispersarse o verterse los residuos inorgánicos no comercializados o no utilizados en la cría. El inadecuado almacenamiento y tratamiento de residuos orgánicos (como podría ser la fermentaciónoelsecado), probablemente acelera su putrefacción, atrayendo insectos o roedores y generando olores desagradables, causantes de conflictos con los vecinos. 
Como los efluentes de criaderos familiares son difíciles de tratar en forma individual, para Castro et al (2002) sería ideal implementar sistemas que solucionen el problema en forma grupal. Sin embargo, esto no ocurre en ninguno de los casos analizados, en los que los efluentes se vierten al sistema de alcantarillado.

9. Estrategias de intervención en busca de soluciones a los problemas

Sin realizar una apología de la crianza animal urbana y periurbana, los municipios deberán encontrar situaciones intermedias entre la represión y el descontrol, teniendo en cuenta que si bien la actividad genera problemas cuando no se desarrolla en forma adecuada, es esta el sustento de poblaciones pobres y vulnerables (Ramírez, 2008).

9.1 Estrategias para enfrentar los problemas legales y políticos

Castro et al (2005) consideran que se deberá elaborar y actualizar la normativa que rige la crianza de animales en los municipios. En algunas ciudades no existe normativa y en otras no se encuentra actualizada. Un buen ejemplo es Montevideo, Uruguay que dispone de marcos legales de 1939 que no se han adaptado a los cambios sufridos por la ciudad ni a las estrategias productivas.

También se debe mejorar la legitimidad política de los criadores y sus organizaciones, favoreciendo la instalación de mesas de diálogo y concertación y promoviendo procesos multi-actorales para definir de manera participativa las estrategias y acciones concretas para el adecuado desarrollo de la actividad.

Un ejemplo para Nasinyama et al (2004) son las reglamentaciones que además de establecer restricciones o permisos, fijen periodos de gracia para mejorar la calidad de sus actividades, como la construcción de planchas en cemento con un adecuado sistema de recolección de orina y excretas que pueden ser utilizadas con otro tipo de fines. Por ejemplo, en Uganda las excretas vacunas con el debido tratamiento, se emplean en el cultivo de hongos comestibles.

\subsection{Estrategias para enfrentar problemas sanitarios}

Esta problemática es crítica e importante, ya que puede incidir en el status sanitario internacional de un país. Para el caso, Uruguay, por medio de vacunación, es hoy un país libre de aftosa, lo que le permite acceder a importantes mercados y vender su producción de carne bovina.

Se deberá establecer un sistema de información sanitaria y elaborar modelos de riesgo de enfermedades de importancia económica y zoonótica. Esto permitirá conocer la situación sanitaria en tiempo real y facilitará la tarea en las épocas de alerta.

Merzthal (2004) propone que también deben ser funcionales los mecanismos de tratamiento y conservación de residuos orgánicos y subproductos, como parte de la gestión integral de los residuos. De esta manera, el tratamiento permitirá lograr la inocuidad del producto final para 
minimizar los riesgos de transmisión de enfermedades, mejorando la viabilidad económica de los criadores pobres. En la actualidad, el sistema más utilizado por los productores es la cocción. Entre sus las ventajas se destaca que puede hacerse a pequeña escala en el criadero y que los materiales para la combustión son de fácil obtención.

En Cienfuegos (Cuba) se encontró que el PLP (Pienso Líquido Procesado) esteriliza los residuos, disminuyendo el riesgo de transmisión de enfermedades casi a cero, y permite grandemente reutilizar tales residuos.

En Montevideo, la Facultad de Veterinaria experimentó un método de tratamiento y conservación de residuos, basado en la tecnología de fermentación controlada, con el cual se puede mantener un producto con su valor nutritivo y $\sin$ alteraciones hasta 45 días. Entre las ventajas que tiene, está su fácil implementación en un criadero y la reutilización de varios residuos.

Finalmente, los productores deben ser capacitados para reconocer las enfermedades de importancia económica y zoonótica, sus manifestaciones y las acciones a tomar en caso de aparición de brotes.

\subsection{Estrategias para enfrentar los problemas ambientales}

Según Castro et al (2005), los principales impactos negativos se generan debido a un manejo inadecuado de los residuos sólidos que ingresan y los residuos sólidos y líquidos que salen. Se debe trabajar, por tanto, en dos aspectos: sistemas de gestión de residuos orgánicos y tratamiento alternativo de efluentes.

\subsection{Tratamiento alternativo de efluentes}

La cantidad de efluentes producidos por los animales supera el uso que se pueda hacer del mismo sin tratar, para abono o combustible. Esta cantidad podrá ser uno de los parámetros que tomen los gobiernos para limitar el número de animales que se deban criar en áreas urbanas y periurbanas.

Para Castro et al (2005), se hace imperativo encontrar alternativas a los métodos tradicionales (lagunas de oxidación) que por costo y necesidad de espacio son inviables para las áreas urbanas. Las pequeñas fosas, el compostaje, el uso de plantas acuáticas y el biogás pueden ser soluciones colectivas adecuadas que deben ser investigadas con mayor detenimiento. Es necesario establecer alianzas con facultades e institutos de investigación para el acompañamiento y la aplicación de investigaciones e innovaciones en forma participativa.

\section{Conclusiones}

Se pudo determinar por medio de experiencias, la manera como es posible relacionar la producción vegetal con la animal en sistemas sostenibles urbanos y periurbanos que generen mayor eficiencia en la solución de problemas relacionados con la seguridad alimentaria.

La producción animal urbana y periurbana contribuye a resolver problemas de seguridad alimentaria entre 
la población más vulnerable que habita en las ciudades, ya que aporta proteínas a la dieta y genera ingresos y empleo.

Entre las limitaciones que tienen los sistemas de cría animal urbanay periurbana están las ambientales, sanitarias, legales y políticas. De ahí la necesidad de que los gobiernos locales establezcan políticas públicas que regulen la actividad, de forma que no se afecte el sistema tradicional campesino de abastecimiento de alimentos a las ciudades.

Las asociaciones y organizaciones de productores hacen que estos sistemas de producción sean más fuertes y se posicionen mejor en las ciudades.

En muchos lugares dedicados a la práctica pecuaria en las ciudades y alrededor de ellas se puede determinar que esta actividad nace como una necesidad de las comunidades para mejorar la calidad de vida; esto hace pensar que es una práctica necesaria y que debe ser tenida en cuenta por los gobiernos locales y nacionales con el fin de generar mayor desarrollo.

Los gobiernos locales y nacionales deben revisar el Plan de ordenamiento territorial para tomar medidas efectivas que reglamenten la cría animal urbana y periurbana, pues al no estar reglamentada impide que organizaciones gubernamentales o privadas generen proyectos de desarrollo.

Mediante los estudios de caso de experiencias positivas alrededor del mundo es posible fortalecer la producción animal sostenible a nivel urbano y periurbano.

\section{Agradecimientos}

El desarrollo de esta investigación ha sido posible debido a la colaboración y el apoyo de la Escuela de Ciencias Agrícolas, Pecuarias y del Medio Ambiente de la Universidad Nacional Abierta y a Distancia (UNAD), CEAD Bogotá.

\section{Referencias}

1) Arce, B., Alegre, J. \& Gordon, P., Escudero, G., Sáenz, J. \& Gordon P. (2007). Crianza de cerdos en zonas urbanas: Diagnóstico y propuesta municipal de sistema de manejo en el distrito de Lurigancho Chosica, Lima (Perú). Programa Cosecha urbana Centro internacional de la papa. En G. Castro (Comp.), Porcicultura urbana y periurbana en ciudades de América latina y el Caribe. Lima, Perú: IPES RUAF.

2) Casanovas, E., Novoa, R., Socorro, A., Parets, E., González, M. \& Liriano, R. (2007).Crianza porcina en la agricultura urbana de Cienfuegos (Cuba). En G. Castro (Comp.), Porcicultura urbana y periurbana en ciudades de América latina y el Caribe. (pp. 19-24) Lima, Perú: IPES RUAF.

3) Castro, G. (2007). Porcicultura urbana y periurbana en ciudades de América Latina y el Caribe. Lima, Perú: IPES RUAF.

4) Castro, G., González, A. \& Lozano, A. (2002). Ciudades productivas. Buscando el equilibrio entre los impactos ambientales y socioeconómicos en los sistemas 
agropecuarios urbanosy periurbanos. En A. Domínguez \& R. Prieto, Perfil ambiental del Uruguay 2002 (pp. 82-97). Montevideo, Uruguay: Nordan Comunidad.

5) Castro, G., Lozano, A., Fernández, G. \& Ronca, F. \& Rodríguez, D. (2005). Agro diversidad y Pobreza Archivos de zootecnia, 54 (206), 205-209. Recuperado de http:// www.uco.es/organiza/servicios/ publica/az/php/imag/web/09_13_51_ AgrobiodiversidadCastro.pdf.

6) Colombia, Instituto de Promoción del desarrollo sostenible. (2006). Módulo de desarrollo técnico. La huerta en la agricultura urbana. Bogotá, Colombia. Recuperado de http://www.ipes.org/index.php.

7) Coto, M. (2005). Acuicultura familiar en Cuba. Revista de agricultura urbana, $A U$ (13), 34-35, Diciembre. La Habana, Cuba. Recuperado de www.actaf.co.cu/revistas/revista au_1-18/rau14/AU14_Cuba.pdf.

8) Drescher, A. (2001). Seguridad alimentaria urbana. ¿Una respuesta a la crisis? Revista de agricultura urbana, AU 1(1), 8-10. La Habana, Cuba. Recuperado de http://www.ipes.org/ image/agriculturaUrbana/documents/ RevEsp1/AUarticulo2.pdf.

9) FAO. (2001). Seguridad alimentaria y conceptos y definición. Disponible en http://www.fao.org/docrep/005/y 4671e/y4671e06.htm.
10)Farmaverde. (2006). Capacitación sobre cultivo y uso de plantas medicinales. Manuscrito no publicado, $42 \mathrm{pp}$.

11)Francia, L. \& Bautista, M. (2005). Crianza de cuyes en zonas urbanas y periurbanas. Revista de agricultura urbana, 2 (14), 5-7. La Habana, Cuba. Recuperado de http://www. actaf.co.cu/revistas/revista_au_1-18/ rau2ruaf2parte1.pdf.

12)Khov, R., Borin, Ch. \& Dareams, S. (2005). Sistemas acuícolas de producción periurbana de alimentos en Phnom Penh. Revista de agricultura urbana, (15), 13-15. La Habana, Cuba. Recuperado de http://www. actaf.co.cu/revistas/revista_au_1-18/ rau2/ruaf2parte3.pdf.

13)Mekasha, Y., Tadesse, M. Tegegne, A. \& Yami, A. (2003). Sistemas urbanos y periurbanos de producción lechera orientados al mercado. Revista de agricultura urbana (10), 21-22. La Habana, Cuba. Recuperado de http:// www.actaf.co.cu/revistas/revista au_1-18/rau2/ruaf2parte7.pdf.

14)Merzthal, G. (2004). Marco normativo y legal para la agricultura urbana. Recuperado de http:// www.bibliotecaverde.wikieco.org/ cuaderno142.pdf.

15)Nasinyama, F. \& Beeck, S. (2004). Limpiando, enverdeciendo y alimentando a la ciudad; iniciativas locales en reciclaje de residuos para la agricultura urbana en Kampala, 
Uganda. Revista de agricultura urbana (10), 36. La Habana, Cuba. Recuperado de http://www.ipes.org/ images/agriculturaUrbana/documents/ revEsp19/14_limpiando.pdf.

16)Nunan, F. (2005). Cría de animales y sustento en Hubli - Dharwad. Revista de agricultura urbana (16), 10-11. La Habana, Cuba. Recuperado de http:// www.actaf.co.cu/revistas/revista au_1-18/rau2/ruaf2parte3.pdf.

17)Piedad, M. (2009). Apicultores urbanos ¿unpasatiempoclandestino? Disponible en http://www.souitues/ souitu/2009/02/18 medioambiente / 1234952247_085179.html.

18)Ramírez, G. (2008). Agricultura Orgánica ( $8^{\mathrm{a}}$ edic). Buga, Colombia: Litobuga.
19)Santandreu, A., Castro, G., Ronca, F. \& Lozano, A. (2006). La cría de cerdos en asentamientos urbanos y periurbanos de Montevideo (Uruguay). En G. Castro (Comp.), Porcicultura urbana y periurbana en ciudades de América latina y el Caribe. Lima, Perú: IPES RUAF.

20)Silvana, M. (2006). Cría de pollos para autoconsumo: Una experiencia en Camilo Aldao. Revista de agricultura urbana. La Habana, Cuba. Recuperado de http://www. actaf.co.cu/revistas/revista_au_118/rau2/ruaf2parte4.pdf

Recibido: 1 de junio de 2011 Aceptado: 15 de septiembre de 2011 